Results: The proteome analysis of SF allowed deepest proteome analysis so far (Proteoforms $>1300$ ) as well as a number of citrullinated sites. The three patient groups could be differentiated by cluster analysis and the occupancy of each modified site calculated. The investigation of the intestinal tissue enabled identification of 223 citrullinated peptides from 121 proteins. Three of the peptides were unique to RA. The list of citrullinated proteins was enriched in extracellular and membrane proteins and included known targets of anticitrullinated protein antibodies (ACPAs). Investigation of collagen-induced arthritis mouse model enabled differential analysis of the proteome as response to treatment and determination of the PTMs associated with this model.

Conclusions: Our deep proteome based analysis of tissue and biofluids have enabled an extended catalogue of citrullinated proteins and sites relevant to improved disease subtyping as well as a source of citrullinated sites for future studies.

References:

[1] Bennike, T.B., Ellingsen, T., Glerup, H., Bonderup, O.K., Carlsen, T.G., Meyer, M.K., Bøgsted, M., Christiansen, G., Birkelund, S., Andersen, V., et al. (2017). Proteome Analysis of Rheumatoid Arthritis Gut Mucosa. J. Proteome Res. 16, 346-354.

[2] Tue Bennike, Kasper B. Lauridsen, Michael Kruse Olesen, Vibeke Andersen, Svend Birkelund and Allan Stensballe (2013). Optimizing the Identification of Citrullinated Peptides by Mass Spectrometry: Utilizing the Inability of Trypsin to Cleave after Citrullinated Amino Acids. Journal of Proteomics \& Bioinformatics 06.

Disclosure of Interest: None declared

DOI: 10.1136/annrheumdis-2017-eular.6934

\section{SAT0021 HUMAN CD4 T CELLS AND SYNOVIAL FIBROBLASTS COOPERATE TO PROMOTE INFLAMMATION IN THE RA SYNOVIAL JOINT}

A. Petrasca ${ }^{1}$, G. Jameson ${ }^{1}$, T. McGarry ${ }^{2}$, D.J. Veale ${ }^{3}$, U. Fearon ${ }^{2,3}$, J.M. Fletcher ${ }^{1,2} .{ }^{1}$ School of Biochemistry and Immunology; ${ }^{2}$ School of Medicine, Trinity Biomedical Sciences Institute, Trinity College Dublin; ${ }^{3}$ Department of Rheumatology, St. Vincent's University Hospital, Dublin, Ireland

Background: Rheumatoid arthritis (RA) is a chronic autoimmune disease characterised by synovial tissue proliferation and degradation of articular cartilage. Activated synovial fibroblasts proliferate and express matrix-degrading proteases, adhesion molecules and proinflammatory cytokines, which contribute to cartilage and joint destruction. Moreover, synovial cell activation correlates with infiltration of inflammatory lymphocytes and monocytes which in turn contribute to synoviocyte activation, thus further exacerbating inflammation.

Objectives: The functional relationship linking fibroblasts and $\mathrm{T}$ lymphocytes in this complex microenvironment has yet to be characterised. Therefore, we established an in vitro model to examine the outcomes of co-culturing activated human CD4 T cells with RA synovial fibroblasts.

Methods: Co-culture assays were carried out using immortalised K4IM RA synovial fibroblasts or synovial fibroblast cells derived from arthroscopy biopsies of RA patients. Human CD4 T cells were stained with a proliferation-tracking dye and co-cultured with pre-seeded synovial fibroblasts for 5 days. The resulting cell cultures and supernatants were examined for proliferation, cytokine production, secretion of matrix metalloproteinases and expression of adhesion molecules.

Results: We found that CD4 T cells and K4IM cells reciprocally induced an increased expression of adhesion molecules ICAM and VCAM. Furthermore, co-culture of CD4 T cells and synovial fibroblasts resulted in proliferation of CD4 T cells expressing increased levels of the proinflammatory cytokines IFN- $\gamma$ and IL17a and RANKL after 5 days. Lastly, co-culture of $T$ cells and synovial fibroblasts resulted in secretion of IL-6, IL-8, IFN- $\gamma$ and IL-17a and matrix metalloproteinases MMP-1 and MMP-3.

Conclusions: These results indicate that CD4 T cells work mutually with synoviocytes to create an inflammatory microenvironment likely to promote joint destruction. Future studies will characterise the role of glucose metabolism in these cells and investigate if metabolism is intrinsically coupled to effector functions in these cells.

Disclosure of Interest: None declared

DOI: 10.1136/annrheumdis-2017-eular.6294

\section{SAT0022 EPIGENOME-WIDE ASSOCIATION STUDY OF RHEUMATOID ARTHRITIS IDENTIFIES DIFFERENTIALLY METHYLATED LOCI IN B CELLS}

A. Julià ${ }^{1}$, D. Absher ${ }^{2}$, M. López-Lasanta ${ }^{1}$, N. Palau ${ }^{1}$, A. Pluma ${ }^{1}$, L. Waite Jones $^{2}$, J.R. Glossop ${ }^{3}$, W.E. Farrell ${ }^{3}$, R.M. Myers ${ }^{2}$, S. Marsal ${ }^{4} .{ }^{1}$ Grup de Recerca de Reumatologia, Vall Hebron Research Institute, Barcelona, Spain; ${ }^{2}$ Absher Lab, HudsonAlpha Institute for Biotechnology, Huntsville, United States; ${ }^{3}$ Keele University, Institute for Science and Technology in Medicine,

Staffordshire, United Kingdom; ${ }^{4}$ Grup de Recerca de Reumatologia, Vall Hebron Research Institute, Alabama, Spain

Background: Epigenetic regulation of immune cell types could be critical for the development and maintenance of autoimmune diseases like Rheumatoid Arthritis (RA). B cells are highly relevant in RA, since patients express autoantibodies and depleting this cell type is a successful therapeutic approach. Epigenetic variation, such as DNA methylation, may mediate the pathogenic activity of $B$ cells.

Objectives: In this study, we performed an epigenome-wide association study (EWAS) for RA with three different replication cohorts, to identify disease-specific alterations in DNA methylation in B cells.

Methods: Genomic methylation in isolated B lymphocytes was assayed on the Illumina HumanMethylation450 BeadChip, assaying $>450,000$ different $\mathrm{CpG}$ sites. Differential methylated positions (DMPs) were identified in a discovery cohort using a single-point analysis using logistic regression, as well as a pathway-level analysis using a newly developed permutation-based method. A discovery cohort of 50 RA patients and 75 healthy controls from Spain was used to identify the most differentially methylated regions after multiple test correction. Using an independent sample of 15 patients and 15 controls from the same population we performed a replication analysis of the most significant CpG sites and pathways. Using an additional case-control sample of 24 individuals from the UK we provided further evidence of association of the DMPs with RA. Finally, in silico datta from a cohort of systemic lupus erythematosus patients (SLE, $n=47)$ and controls $(n=56)$ from the US, we tested the association of the associated DMPs.

Results: A total of $64 \mathrm{CpG}$ sites were found to be differentially methylated in RA patients compared to controls in the discovery stage after multiple test correction $(q<0.05)$. Six biological pathways were also differentially methylated in RA B cells. Analysis of these epigenetic changes in the independent Spain cohort replicated the association of $10 \mathrm{CpG}$ sites located on 8 genes and 2 intergenic regions. Differential methylation at the $C B L$ signaling pathway was also replicated. Using the UK case-control cohort, association between RA risk and methylation levels at $C D 1 C\left(P=2.26 \times 10^{-9}\right)$ and TNFSF10 $\left(P=1.67 \times 10^{-8}\right)$ loci was further validated. Most of the replicated DMPs associated with RA were also found to be associated with differential methylation in SLE B cells.

Conclusions: Our results highlight genes that may drive the pathogenic activity of $B$ cells in RA and suggest shared methylation patterns with SLE.

Disclosure of Interest: None declared

DOI: 10.1136/annrheumdis-2017-eular.2338

\section{SAT0023 DIRECT-ACTING ANTIVIRAL-BASED THERAPY RESTORES IMMUNE TOLERANCE IN HEPATITIS C-INDUCED CRYOGLOBULINEMIA VASCULITIS}

C. Comarmond ${ }^{1}$, M. Garrido ${ }^{2}$, A.-C. Desbois ${ }^{2}$, M. $^{2}$ Costopoulos $^{3}$, M. Le Garff-Tavernier $^{3}$, S.N. Si Ahmed ${ }^{4}$, L. Alric ${ }^{5}$, H. Fontaine $^{6}$, B. Bellier ${ }^{7}$, A. Maciejewski ${ }^{7}$, M. Rosenzwajg ${ }^{7}$, D. Klatzmann ${ }^{7}$, L. Musset ${ }^{8}$, T. Poynard ${ }^{9}$, P. Cacoub ${ }^{10}$, D. Saadoun ${ }^{10} .{ }^{1}$ Internal Medicine and Clinical Immunology; ${ }^{2}$ INSERM, UMR s 959, FRE3632, Hôpital Pitié-Salpêtrière; ${ }^{3}$ Biological Hematology, Groupe Hospitalier Pitié-Salpêtrière, Paris; ${ }^{4}$ Hepatology, Hôpital d'Orléans, Orléans; ${ }^{5}$ Internal Medicine, Centre hospitalier universitaire Purpan, Toulouse; ${ }^{6}$ Hepatology, CHU Cochin; ${ }^{7}$ INSERM, UMR s 959, FRE3632; ${ }^{8}$ Immunology, UF d'Immunochimie et d'autoimmunité; ${ }^{9}$ Hepatology; ${ }^{10}$ Internal Medicine and Clinical Immunology, CHU Pitié-Salpêtrière, Paris, France

Objectives: Interferon-free direct-acting antiviral (DAA)-based therapy has proven to be very effective in patients with hepatitis $C$ virus-cryoglobulinemia vasculitis (HCV-CV). However, their mechanisms of action and their effects on cellular immunity remain poorly defined.

Methods: $27 \mathrm{HCV}-\mathrm{CV}$ patients treated with DAA therapy, 12 healthy donors (HD) and $12 \mathrm{HCV}$ were included. We investigated the effects of DAA-based therapy on cellular and cytokine abnormalities in HCV-CV patients by flow cytometry, cytokine Multiplex and enzyme-linked immunosorbent assay.

Results: Compared with HD and HCV, pre-DAA abnormalities in HCV-CV patients included a decreased percentage of $\mathrm{CD} 4^{+} \mathrm{CD} 25^{\text {hi }} \mathrm{FoxP}^{+}$regulatory $T$ cells $(P<0.01)$ with increases in $\operatorname{lgM}^{+} C D 21^{- \text {llow }}$ memory $B$ cells $(P<0.05)$, $\mathrm{CD}^{+} \mathrm{IFN} \gamma^{+}(\mathrm{P}<0.01), \mathrm{CD}^{+} \mathrm{IL} 17 \mathrm{~A}^{+}(\mathrm{P}<0.01)$ and $\mathrm{CD} 4^{+} \mathrm{CXCR5} 5^{+} \mathrm{IL} 21^{+}$follicular helper $T$ cells $(T f h)(P<0.01)$. IgM ${ }^{+} C D 21^{-1 / l o w}$ memory $B$ cells were negatively correlated with regulatory $T$ cells (Tregs) $(\mathrm{P}=0.03)$, and positively correlated with Tfh $(P=0.03)$ and serum cryoglobulin levels $(P=0.01)$. DAA-based therapy was associated with an increase in Tregs frequency $(1.5 \% \pm 0.18 \%$ versus $2.1 \%$ $\pm 0.18 \%$ ), and decreased $\operatorname{lgM}{ }^{+} \mathrm{CD} 21^{-1 / 0 w}$ memory $\mathrm{B}$ cells and Tfh percentage $(35.7 \% \pm 6.1 \%$ versus $14.9 \% \pm 3.8 \%$, and $12 \% \pm 1.3 \%$ versus $8 \% \pm 0.9 \%$, respectively). B lymphocyte stimulator receptor 3 and programmed death-ligand 1 staining expression on B cells increased in HCV-CV after DAA-based therapy (MFI $37 \pm 2.4$ versus $47 \pm 2.6, P<0.01$; and $29 \pm 7.3$ versus $48 \pm 9.3, P<0.05$ respectively). Conclusions: Our results indicate that DAA-based therapy effectively normalizes many of the disturbances in peripheral B and T lymphocyte homeostasis of HCV-CV patients.

Disclosure of Interest: None declared

DOI: 10.1136/annrheumdis-2017-eular.3911

\section{SAT0024 ALTERATIONS OF PERIPHERAL BLOOD B-CELL SUBSETS IN EARLY RHEUMATOID ARTHRITIS}

E. Suponitskava, A. Avdeeva, A.A. Aleksankin, E. Gerasimova, E. Aleksandrova, T. Popkova, A.N. Novikov, D.K. Karateev. Institute of Rheumatology, Moscow, Russian Federation

Background: Alterations of B-cell subpopulation have been described in the 
peripheral blood of rheumatoid arthritis (RA) patients (pts), but differences between B-cell subsets distributions in early and late onset RA (LORA) are still unclear $[1,2]$.

Objectives: To examine B-cell subsets in peripheral blood of healthy donors, early (disease duration $<6$ months) RA (ERA) and LORA pts by flow cytometry, and to analyze the association between B-cell subsets and RA activity.

Methods: 24 active ERA pts (20F/4M); median age 54 years (range) (38-64); disease duration 5 months (4-6); DAS28 score 5.2 (4.7-5.9); RF+75\%, ACCP+ $87.5 \%$ and 20 LORA pts (16F/4M); median age 58 years (range) (44-62); disease duration 12 years (4.5-17.5); DAS28 score 5.3 (4.6-6.2); both RF+ and ACCP+ $80 \%$, were assessed for B-cell subpopulations and laboratory data: ESR, CRP. LORA pts were treated with DMARDs (anti-TNF- $\alpha$, Methotrexate), glucocorticoids, and NSAIDS; ERA pts received NSAIDS only. CD19+B cells, memory B cells (CD19+CD27-), non-switched memory B cells (CD19+lgD+CD27+), switched memory B cells (CD19+lgD-CD27+), naïve (CD19+lgD+CD27-), double-negative (CD19+lgD-CD27-), transitional (CD19+lgD+CD10+CD38++CD27-) B cells, and plasmablasts (CD19+CD38+++lgD-CD27+CD20-) were analyzed using multicolor flow cytometry.

Results: The median percentages (range) of non-switched memory B cells (CD19+lgD+CD27+) were lower in ERA and LORA RA cohorts compared to donors: $3.0 \%(1.6-5.5)$ and $6.2 \%(2.4-10.2)$ vs $7.4 \%$ (3.7-11.1), respectively, $\mathrm{p}<0.05$ for both cases. In LORA pts, the median percentages (range) and absolute numbers of switched memory $B$ cells (CD19+lgD+CD27-) were higher compared to both ERA pts and donors: $24,1 \%(14,0-42.3)$ vs $8,3 \%(4.4-12.9)$ and $12.8 \%(9.3-17.0)$; and $0.03(0.02-0.05)$ vs $0.01(0.006-0.02)$ and 0.02 (0.01-0.04), respectively; $p<0.05$ for all cases. The median percentages of naive $B$ cells $(C D 19+\lg D+C D 27-)$ were significantly higher in ERA pts compared to LORA pts and healthy donors: $75.3 \%(69.7-83.6)$ vs $54.3 \%(39.7-69.1)$ and $64.7 \%$ (57.6-72.4), respectively, $p<0.05$ for both cases.

In ERA pts, we found a significant correlation between absolute numbers of non-switched memory $B$ cells (CD19+lgD+CD27+), RF and anti-CCP: $r=0.50$ and 0.45 , respectively, $p<0.05$ for both cases. In LORA pts, the percentage of memory $B$ cells $(C D 19+C D 27+)$ correlated positively with $E S R(r=0.58), p<0.05$; a positive association was observed between the absolute numbers of switched memory $B$ cells $(C D 19+\operatorname{lgD}-C D 27+), C R P(r=0.48)$ and $R F(r=0.49), p<0.05$ for all cases.

Conclusions: in ERA pts, immunophenotyping showed increased frequencies of naïve $B$ cells and decreased frequencies and absolute numbers of switched memory B cells compared to LORA cohort.

References:

[1] Fedele et al. BMC Immunology 2014, 15:28. doi: 10.1186/s12865-014-00281. Memory B cell subsets and plasmablasts are lower in early than in long-standing Rheumatoid Arthritis.

[2] Moura RA et al. Rheumatology 2010 Jun;49(6):1082-92. doi: 10.1093/ rheumatology/keq029. Alterations on peripheral blood B-cell subpopulations in very early arthritis patients.

Disclosure of Interest: None declared

DOI: 10.1136/annrheumdis-2017-eular.1533

\section{SAT0025 MIR 106A, MIR 19A-B, MIR 20A AND MIR21A REGULATE V $\gamma 9$ V $\delta 2$ FUNCTIONS PARTICIPATING IN THE INFLAMMATORY RESPONSES OCCURRING IN RHEUMATOID ARTHRITIS}

G. Guggino ${ }^{1}$, F. Ciccia ${ }^{1}$, V. Orlando ${ }^{2}$, L. SAieva ${ }^{2}$, P. Ruscitti ${ }^{3}$, P. Cipriani ${ }^{3}$, I. Chalcarz ${ }^{4}$, R. Alessandro ${ }^{4}$, R. Giacomelli ${ }^{3}$, F. Dieli $^{4}$, N. Caccamo ${ }^{4}$, G. Triolo ${ }^{4}$. ${ }^{1}$ DIBIMIS; ${ }^{2}$ DIBIME, University of Palermo, palermo; ${ }^{3}$ University of L'Aquila, L'Aquila; ${ }^{4}$ University of Palermo, Palermo, Italy

Background: miRNAs are non-coding RNAs which have significant roles in regulating gene expression. The miR17-92 cluster appears to be a key factor in the inflammatory pathways activated during RA.

Objectives: In this study we aimed to evaluate miR17-92 expression and functions in $\gamma \delta \mathrm{T}$ cell subsets in RA patients, $\gamma \delta \mathrm{T}$ cells, in fact produce proinflammatory cytokines such as IFN-g, IL-6 and IL-8 that may contribute to the inflammatory responses in RA.

Methods: Heparinized peripheral blood from 10 early RA untreated patients and 10 healthy donors was obtained for this study. Polyclonal $\mathrm{V} \gamma 9 \mathrm{~V} \delta 2 \mathrm{~T}$ cell lines were generated first by magnetic isolation followed by sorting (FACSAria) and further analysed by flow cytometry to define their phenotype and their pattern of cytokine production. Expression analysis of miRNA17-92 cluster was performerd by RT-PCR and after identification of relevant miRNA involved in RA, mRNA expression of miRNA target genes was studied.

Results: A remarkable change in the distribution of $\mathrm{V} \gamma 9 \mathrm{~V} \delta 2 \mathrm{~T}$ cell functional subsets with an expansion of effector subsets and a reduction of naïve cells, was observed in the peripheral blood of RA patients, as compared to healthy donors, which were accompanied by modifications in proinflammatory cytokine expression. Particularly, TEM (effector memory) and TEMRA (effector memory terminally differentiated) cells resulted the major souce of IL-6 and IL-8. A significant correlation between miRNA expression and levels of IL- 6 and IL-8 was found. The comparative analysis of miRNA expression among $V \gamma \gamma \mathrm{V} \delta 2 \mathrm{~T}$ cell subsets, between RA patients and healthy controls showed a downregulation of miR106a-5p and miR20a-5p and an upregulation of miR $21 \mathrm{a}-5 \mathrm{p}$ among $\mathrm{V} \gamma \mathrm{V} \mathrm{V} \delta 2 \mathrm{~T}_{\mathrm{EM}}$ cells; a downregulation of miR19-3p among $V_{\gamma} 9 \mathrm{~V} \delta 2 \mathrm{~T}_{E M}$ and $\mathrm{TC}_{\mathrm{M}}$ (central memory) cells was also found. This miRNA expression regulated IL- 8 and IL- 6 gene transcription contributing to the survival of the pro-inflammatory pool reducing the expression of the PDCD4 gene.

Conclusions: Our results provide evidence of a role of miR106a, miR19-3p, $20 \mathrm{a}$ and $21 \mathrm{a}$ in the regulation of $\mathrm{V} \gamma 9 \mathrm{~V} \delta 2 \mathrm{~T}$ cells function in RA patients and suggest the possibility that the miR17-92 family and $\gamma \delta$ T cells participate to the pathogenesis of RA.

Disclosure of Interest: None declared

DOI: 10.1136/annrheumdis-2017-eular.5698

\section{SAT0026 SIGNALLING THROUGH INSULIN-LIKE GROWTH FACTOR 1 RECEPTOR CONTRIBUTES TO IL-6 PRODUCTION AND SUPPORTS T CELL DEPENDENT INFLAMMATION IN RHEUMATOID ARTHRITIS}

M. Erlandsson ${ }^{1}$, M. Nadali ${ }^{2}$, S. Töyrä Silfverswärd ${ }^{2}$, M.N. Svensson ${ }^{2}$, I.-M. Jonsson ${ }^{2}$, K.M. Andersson ${ }^{3}$, M.I. Bokarewa ${ }^{2}{ }^{1}{ }^{1}$ Rheumatology and Inflammation Research; ${ }^{2}$ Dept of Rheumatology and Inflammation Research; ${ }^{3}$ Dept. of Rheumatology and Inflammation Research, Gothenburg University, Gothenburg, Sweden

Background: Insulin-like growth factor (IGF) 1 receptor is essential for cell energy metabolism. It plays a key role linking glucose and lipid metabolism of active cells and may turn pathogenic in uncontrolled tissue growth in tumours. In rheumatoid arthritis (RA), the IGF-1R signalling is activated supporting expansion of the inflamed synovia.

Objectives: The aim of the present study was to understand the place of IGF-1R expression and signalling for development of adaptive immune responses in the setting of prospective RA cohort and in experimental arthritis.

Methods: Clinical associations of IGF1R expression in leukocytes of the peripheral blood were studied in 84 female RA patients with mean disease duration 8 years, all treated with methotrexate. Consequences of the IGF1R signalling inhibition for arthritis were studied in mBSA immunised Balb/c mice treated with NT157 compound promoting degradation of the insulin receptor substrates IRS-1/2.

Results: In RA patients, high expression of IGF1R in leukocytes was associated with systemic inflammation as verified by higher transcription factor NF-kB $(p=0.033)$, serum levels of IL-6 $(p=0.006)$ and erythrocyte sedimentation rate $(p=0.003)$, and higher pain perception (VAS, $p=0.019)$. Additionally, women with high IGF1R were often in need of combined DMARD treatment $(\mathrm{p}=0.045)$. Phosphorylated IGF1R and STAT3 enrich T cells infiltrate in RA synovia. Treatment with NT157 rendered no metabolic consequences to mouse body weight and serum glucose levels. NT157 inhibited the phosphorylation of IGF1R and STAT3 in synovia, and alleviated arthritis and erosions in mice. It also reduced IGF1R+ $\mathrm{T}$ cell population in spleen and despaired ERK and Akt signalling. This limited the ability of mBSA-specific T cells to produce IL-6 ( $p=0.013)$, IFNg $(p=0.024)$ and IL-17 ( $p=0.0097)$. The reduction of IL- 6 and IL-17 levels was associated with changed RoRgt/FoxP3 balance.

Conclusions: IGF1R signalling contributes to severe RA by triggering $T$ cell dependent inflammation in arthritis. Inhibition of IGF1R on the level of insulin receptor substrates alleviates arthritis by restricting IL- 6 dependent formation of Th17 cells and may open for new treatment strategies in RA.

Disclosure of Interest: None declared

DOI: 10.1136/annrheumdis-2017-eular.6191

\section{SAT0027 IMMUNOMODULATORY EFFECTS OF MGD010, A DART ${ }^{\circledR}$ MOLECULE TARGETING HUMAN B-CELL CD32B AND CD79B}

W. Chen, S. Shankar, J. Lohr, X.-T. Yao, H. Li, X. Chen, J. Muth, N. Gal-Edd E. Bonvini, S. Johnson, N. Pandya, P.A. Moore, J. Wigginton. Macrogenics, INC., Rockville, United States

Background: B-cell targeted therapeutics have proven efficacious in the treatment of autoimmune diseases, generating impetus to develop alternate strategies that provide activity with rapid onset and no B-cell depletion. To this end, MGD010, a bispecifc DART ${ }^{\circledR}$ molecule, was designed to exploit the inhibitory function of the checkpoint molecule, CD32B, (Fc $\gamma$ Rllb) via its simultaneous co-ligation with the BCR component, CD79B. Initial phase 1 studies performed in healthy subjects demonstrated MGD010 was well tolerated in a single-dose, dose escalation to up to $10 \mathrm{mg} / \mathrm{kg}$, with no evidence of peripheral B-cell activation or depletion. MGD010 exhibited pharmacokinetic properties of an antibody-based molecule, with dose-dependent B-cell occupancy saturating at $>1 \mathrm{mg} / \mathrm{kg}$ and diminished propensity for ex-vivo BCR-induced B-cell activation (1). To further understand the potential for MGD010 to modulate ongoing immune responses, the impact of MGD010 on the immune response to Hepatitis A (HAV) vaccination, a T-cell dependent neo-antigen challenge, was evaluated in normal healthy subjects.

Objectives: To determine effects of MGD010 on humoral and cellular immune responses in normal healthy volunteers.

Methods: This was an open label, placebo controlled cohort-expansion in normal healthy volunteers. Subjects ( $n=8$ per group) were randomly assigned to receive an IV administration of a single dose of 3 or $10 \mathrm{mg} / \mathrm{kg}$ MGD010 or placebo. All subjects received a single intramuscular dose of hepatitis $A$ vaccine (HAV) $(1.0 \mathrm{~mL}$ or $\sim 50 \mathrm{U}) 24$ hours after administration of MGD010 or placebo. Subjects were monitored for adverse events for 56 days after administration of drug or placebo. Analyses included peripheral lymphocyte 